TO APPEAR IN The Astrophysical Journal.

Preprint typeset using LATEX style emulateapj v. 5/2/11

\title{
ALIGNMENTS OF BLACK HOLES WITH THEIR WARPED ACCRETION DISKS AND EPISODIC LIFETIMES OF ACTIVE GALACTIC NUCLEI
}

\author{
YAN-RONG LI ${ }^{1}$, JIAN-MIN WANG $^{1,2}$, CHENG CHENG ${ }^{2}$, AND JIE QIU ${ }^{1}$ \\ To appear in The Astrophysical Journal.
}

\begin{abstract}
Warped accretion disks have attracted intensive attention because of their critical role on shaping the spin of supermassive massive black holes (SMBHs) through the Bardeen-Petterson effect, a general relativistic effect that leads to final alignments or anti-alignments between black holes and warped accretion disks. We study such alignment processes by explicitly taking into account the finite sizes of accretion disks and the episodic lifetimes of AGNs that delineate the duration of gas fueling onto accretion disks. We employ an approximate global model to simulate the evolution of accretion disks, allowing to determine the gravitomagnetic torque that drives the alignments in a quite simple way. We then track down the evolutionary paths for mass and spin of black holes both in a single activity episode and over a series of episodes. Given with randomly and isotropically oriented gas fueling over episodes, we calculate the spin evolution with different episodic lifetimes and find that it is quite sensitive to the lifetimes. We therefore propose that spin distribution of SMBHs can place constraints on the episodic lifetimes of AGNs and vice versa. Applications of our results on the observed spin distributions of SMBHs and the observed episodic lifetimes of AGNs are discussed, although both the measurements at present are yet ambiguous to draw a firm conclusion. Our prescription can be easily incorporated into semi-analytic models for black hole growth and spin evolution.
\end{abstract}

Subject headings: accretion, accretion disks — black hole physics — galaxies: active — quasars: general

\section{INTRODUCTION}

Accretion disks surrounding supermassive black holes (SMBHs) are believed to be the source engines of enormous radiative power of active galactic nuclei (AGNs) in the universe (e.g., Salpeter 1964; Lynden-Bell 1969). The shapes of accretion disks-flat or warped-depend on the orientation of the angular momentum of gas fueling from the circumnuclear region with respect to the rotating orientation of the central black hole. A wide variety of evidence have indicated that in many circumstances these two orientations are misaligned.

First, the orientation of AGNs seems uncorrelated with that of their host galaxies, well established by observations (Kinney et al. 2000; Schmitt et al. 2003; Gallimore et al. 2006; Muñoz Marín et al. 2007; Shen et al.|2010; Lagos et al. 2011) and also confirmed by numerical simulations (e.g., Hopkins et al. 2012 and references therein). This suggests that gas channeled onto the central SMBH either loses the memory of its initial direction in the host galaxy or comes from outside the galaxy (e.g., Dubois et al. 2012; Nayakshin et al. 2012; Gaspari et al. (2013), most likely due to galaxy mergers (Kendall et al. 2003). Secondly, the current hierarchical framework of galaxy formation and evolution predicts that repeat galaxy mergers, in addition to secular evolution, trigger SMBH activities (e.g., Benson \& Bower 2010) and gas accretion plausibly proceeds at episodic and random phases (in particular for minor mergers; e.g., King \& Pringle 2006; Wang et al. 2006). Thirdly, recent studies on SMBH spin through quantifying the radiative efficiency of AGN populations under the thin accretion disk model showed that SMBH spin undergoes a decline with cosmic time sine $z \sim 2$ Wang et al. 2009; Li et al. 2012), potentially implying that

\footnotetext{
1 Key Laboratory for Particle Astrophysics, Institute of High Energy Physics, Chinese Academy of Sciences, 19B Yuquan Road, Beijing 100049, China; liyanrong@ mail.ihep.ac.cn

2 National Astronomical Observatories of China, Chinese Academy of Sciences, 20A Datun Road, Beijing 100020, China
}

(partially) random accretion takes place (King et al. 2008). Such evolutionary behavior has also been reproduced by Volonteri et al. (2013) in their semi-analytic model of SMBH growth and evolution (see their Figure 10).

Once gas fueling is inclined with respect to the central spinning black hole, a warped accretion disk forms inevitably. The Lense-Thirring torque arising from the frame dragging effect will lead to precessions of the inclined accretion disk and the black hole around each other. Detailed dynamics of warped accretion disks has been studied extensively through theoretical analysis (e.g., Pringle 1992; Papaloizou \& Lin 1995; Ogilvie 1999; (Lubow et al. 2002) and numerical simulations (e.g., Nelson \& Papaloizou 1999, 2000; Lodato \& Pringle 2006, 2007; Fragile 2009; Zhuravlev et al. 2014). The intensive applications of warped accretion disks lie at their critical role on shaping the spin of the central SMBHs through the Bardeen-Petterson effect (Bardeen \& Petterson 1975). In this effect, the presence of strong viscosity, a most like case for normal AGNs, damps out the differential Lense-Thirring precession and induce the inner portion of the disk to align or anti-align with the spin axis of the hole. Accompanied with warp propagation, the entire disk will tend to align or antialign with the hole (Scheuer \& Feiler 1996; King et al. 2005; Martin et al. (2007).

Previous studies on the alignments between black holes and their warped accretion disks mainly focus on two aspects. The first assumes that accretion disks have infinite extensions and constant profiles. Black holes are always driven toward the disks and the black hole-disk systems end up alignments (Scheuer \& Feiler 1996; Martin et al. 2007; Perego et al. 2009). On the contrary, the second assumes that accretion disks are finite in both size and mass and the total angular momentum of the systems is conserved King et al. 2005, 2008), which means that there is no gas supply to the disks. Such systems can end up a configuration of either alignment or anti-alignment, depending on the initial incli- 
nations and the ratios of the angular momenta between disks and black holes (King et al. 2005).

In reality, it is well known that accretion disks are truncated by their own self-gravity in the outer regions where it becomes dominated over the gravity from black holes (e.g., Paczynski 1978; Shlosman \& Begelman 1987; Collin \& Zahn 1999). Outside the truncation radius, the disks fragment into dense clumps and finally turn to be a hotbed of star formations (e.g., Goodman 2003; Levin 2007; Wang et al. 2010; Jiang \& Goodman 2011). It comes naturally that the stellar radiation and supernova explosions feedback to the ambient gas, exciting turbulence that acts to transfer the angular momentum and leads to gas supply to the accretion disks from the further outer regions (Wada \& Norman 2002; Kawakatu \& Wada 2008; Kumar \& Johnson 2010; Wang et al. 2010). Therefore, to appropriately study warped accretion disks, it is very practical to take into account both the finite sizes limited by the self-gravity and the plausible gas feeding at the outer boundaries (see also the discussions of Li et al. 2013). The lifetime that the gas feeding lasts for, in principle, can be determined from the observed episodic lifetime of AGNs, for which there are several techniques, although the results are still primitive (see Martini 2004 for a review).

In this paper, we revisit the alignment processes of black holes with their warped accretion disks by including the finite sizes of accretion disks and the continuous gas feeding controlled by the episodic lifetimes of AGNs. For the convenience of numerical implementation, we introduce a parameter "the lifetime of gas fueling" to describe the duration of gas supply. We employ an approximate global model following Kumar et al. (2008) to describe the accretion process. This enables us to study the alignments of finite-size accretion disks in a quite simply way, avoiding being immersed in the time-consuming numerical simulations on the evolution of accretion disks.

The paper is organized as follows. Section 2 describes the properties of warped accretion disks and Section 3 derives the basic evolution equations for the black hole-accretion disk system. In Section 4, we present the results for alignments/anti-alignments and spin evolution in a single accretion event and over a series of events. We then show the implications of our calculations for spin distribution and episodic lifetimes of AGNs in Section 5. Discussions on plausible caveats of our calculations and conclusions are given in Section 6.

\section{PROPERTIES OF WARPED ACCRETION DISKS}

In the weak-field limit, the frame-dragging effect due to a spinning black hole exerts a Lense-Thirring torque on each annulus of the accretion disk (Bardeen \& Petterson 1975). By denoting the angular momentum of the annulus at a radius $R$ as $\boldsymbol{L}(R)$ and that of the black hole as $\boldsymbol{J}_{\mathrm{h}}$, the Lense-Thirring torque reads

$$
\boldsymbol{t}_{\mathrm{LT}}=\frac{2 G}{c^{2}} \frac{\boldsymbol{J}_{\mathrm{h}} \times \boldsymbol{L}(R)}{R^{3}},
$$

where $G$ is the gravitational constant and $c$ is the speed of light. This Lense-Thirring torque leads the annuli to precess around the rotating axis of the black hole at a rate of $2 G J_{\mathrm{h}} / c^{2} R^{3}$. Since such differential precessing rate rapidly declines with radius as $R^{-3}$, the inner region of the accretion disk is intensely affected in the beginning and gradually warps propagate outwards over the entire disk. Detailed theoretical analysis showed that the dynamics of warped accretion disks is characterized into two regimes depending on the importance of the pressure force and viscous force (Papaloizou \& Lin 1995). If adopting the standard $\alpha$ prescription of viscosity, warps propagate as bending waves for inviscid or sufficiently thick disks $(\alpha<H / R)$, whereas propagate in a diffusive fashion for strongly viscous disks $(\alpha>H / R)$. Here $H$ is the semi-thickness of disks. For simplicity, we only consider thin and viscous accretion disks with Keplerian rotation, in which $\alpha>H / R$ is generally satisfied. This is the most likely case for accretion disks in AGNs with the Eddington ratio at around 0.01-1, the largest population in AGN surveys (e.g., McLure \& Dunlop 2004; Shen et al. 2008).

In the presence of high viscosity, the differential precess in the inner disk will be damped out rapidly, giving rising to an inner flat region (so called Bardeen-Petterson effect; Bardeen \& Petterson 1975). This region extends to the radius where the precessing timescale is comparable with the warp-diffusion timescale, The precessing time scale reads $c^{2} R^{3} / 2 G J_{\mathrm{h}}$ and the warp-diffusion time scale reads $R^{2} / \nu_{2}$, leading to the warp radius as

$$
R_{\mathrm{w}} \sim \frac{2 G J_{\mathrm{h}}}{\nu_{2} c^{2}}
$$

where $\nu_{2}$ is the vertical shear viscosity governing the warp diffusion through the accretion disks.

Integrating over all the annuli yields the total torque exerted on the accretion disk

$$
\boldsymbol{T}_{\mathrm{LT}}=\frac{4 \pi G}{c^{2}} \boldsymbol{J}_{\mathrm{h}} \times \int \frac{\boldsymbol{L}(R)}{R^{2}} d R .
$$

As a reaction, the black hole, in the meantime, suffers an equal but opposite torque that cause its angular momentum to process as well. By taking into account the angular momentum carried by mass accretion, the angular momentum of the black hole evolves following

$$
\frac{d \boldsymbol{J}_{\mathrm{h}}}{d t}=\dot{M}_{\mathrm{h}} \ell_{\mathrm{ms}} \hat{\boldsymbol{J}}_{\mathrm{h}}-\boldsymbol{T}_{\mathrm{LT}}
$$

where $\dot{M}_{\mathrm{h}}$ is the mass accretion rate onto the black hole and $\ell_{\mathrm{ms}}$ is the specific angular momentum at the marginal stable orbit $R_{\mathrm{ms}}$. Hereafter a hat symbol on top of a vector represents the corresponding direction vector.

Similarly, the angular momentum of disks changes with time as

$$
\frac{d \boldsymbol{J}_{\mathrm{d}}}{d t}=-\dot{M}_{\mathrm{h}} \ell_{\mathrm{ms}} \hat{\boldsymbol{J}}_{\mathrm{h}}+\boldsymbol{T}_{\mathrm{LT}}+\dot{\boldsymbol{J}}_{\mathrm{f}}
$$

where $\boldsymbol{J}_{\mathrm{d}}$ is angular momentum summed over all the annuli and $\dot{\boldsymbol{J}}_{\mathrm{f}}$ is angular momentum change due to gas fueling. Note that $\boldsymbol{T}_{\mathrm{LT}}$ is always orthogonal to $\boldsymbol{J}_{\mathrm{h}}$, therefore, it can be expressed in a form of (King et al. 2005)

$$
\boldsymbol{T}_{\mathrm{LT}}=K_{1} \hat{\boldsymbol{J}}_{\mathrm{h}} \times \hat{\boldsymbol{J}}_{\mathrm{d}}+K_{2} \hat{\boldsymbol{J}}_{\mathrm{h}} \times\left(\hat{\boldsymbol{J}}_{\mathrm{h}} \times \hat{\boldsymbol{J}}_{\mathrm{d}}\right),
$$

where the first term in the right hand side corresponds to the precession and the second term corresponds to change in the angle between the hole and the disk. We keep in mind that both $K_{1}$ and $K_{2}$ are generally positive as shown in our previous numerical analysis (Li et al. 2013).

Considering that the inner region of the disk is aligned with the black hole and the Lense-Thirring torque drops off rapidly with radius, the major contribution to $\boldsymbol{T}_{\mathrm{LT}}$ in Equation (3) 
comes from the integral around the warp radius $R_{\mathrm{w}}$. We therefore make an approximate for $K_{2}$ as

$$
K_{2} \approx \frac{2 \pi G}{c^{2}} \frac{J_{\mathrm{h}} L\left(R_{\mathrm{w}}\right)}{R_{\mathrm{w}}} .
$$

Below we will show that such an approximation of $K_{2}$ yields results that exactly agree with the previous analytical studies for small warps (Scheuer \& Feiler 1996; Martin et al. 2007). Note that $K_{1}$ only governs the precession of the black holedisk system and therefore is not important for the present purpose.

\section{BASIC EVOLUTION EQUATIONS}

\subsection{Accretion Disks and the Inclination Angles}

From above equations, we can now determine the evolution of the angular momenta $J_{\mathrm{h}}$ and $J_{\mathrm{d}}$ and the angle $\theta$ between the black hole and the accretion disk. We first define a lifetime of gas fueling $\Delta T_{\mathrm{f}}$ as during which the accretion disk is continuously fed at a mass rate limited by the Eddington rate $\dot{M}_{\text {Edd }}=L_{\text {Edd }} / \eta c^{2}=2.2 M_{8} \eta_{-1}^{-1} M_{\odot} \mathrm{yr}^{-1}$, where $L_{\text {Edd }}$ is the Eddington luminosity, $M_{8}=M_{\mathrm{h}} / 10^{8} M_{\odot}$, and $\eta=0.1 \eta_{-1}$ is the radiative efficiency for mass accretion (see below Section 3.2 for definition).

Note again the inner region of the disk is aligned with the black hole. Multiplying $\hat{\boldsymbol{J}}_{\mathrm{h}}$ to Equation (4) yields

$$
\frac{d J_{\mathrm{h}}}{d t}=\dot{M}_{\mathrm{h}} \ell_{\mathrm{ms}},
$$

and multiplying $\hat{\boldsymbol{J}}_{\mathrm{d}}$ to Equation (5) yields

$$
\frac{d J_{\mathrm{d}}}{d t}=-\dot{M}_{\mathrm{h}} \ell_{\mathrm{ms}} \cos \theta-K_{2} \sin ^{2} \theta+\dot{J_{\mathrm{f}}} \cos \theta,
$$

where we assume that the angular momentum of gas fueling is aligned with $\boldsymbol{J}_{\mathrm{d}}$. The angle between the black hole and the accretion disk changes with time as

$$
J_{\mathrm{d}} J_{\mathrm{h}} \frac{d \cos \theta}{d t}=\frac{d \boldsymbol{J}_{\mathrm{h}} \cdot \boldsymbol{J}_{\mathrm{d}}}{d t}-J_{\mathrm{h}} \cos \theta \frac{d J_{\mathrm{d}}}{d t}-J_{\mathrm{d}} \cos \theta \frac{d J_{\mathrm{h}}}{d t} .
$$

We distinguish the evolution of $\theta$ into two phases as below.

\subsubsection{Phase I: Gas Fueling}

During the phase with gas fueling, the accretion disk is continuously fed so that it maintains a preferred steady angular momentum distribution. As a result, one shall expect that the term due to gas fueling cancels out the other terms in Equations (5) and (9) and $\boldsymbol{J}_{\mathrm{d}}$ and its magnitude $J_{\mathrm{d}}$ remain somewhat constant, i.e., $d \boldsymbol{J}_{\mathrm{d}} / d t=0$ and $d J_{\mathrm{d}} / d t=0$. With Equations (3) and (7), we can simplify Equation (10) into

$$
\frac{d \cos \theta}{d t}=\frac{K_{2}}{J_{\mathrm{h}}} \sin ^{2} \theta .
$$

Since $K_{2}$ is positive, $\theta$ always tends to decrease towards zero regardless of its initial value, i.e., alignment between the disk and the black hole (see also Scheuer \& Feiler 1996; Martin et al. 2007; Perego et al. 2009).

In this phase, as the disk maintains its profile steadily, $K_{2}$ can be deemed to be a constant. There exists an analytic solution for Equation

$$
\frac{1-\cos \theta}{1+\cos \theta}=\frac{1-\cos \theta_{0}}{1+\cos \theta_{0}} \exp \left(-\frac{2 t}{T}\right),
$$

where $\theta_{0}$ is the initial value of $\theta(t)$ at $t=0$ and $T=J_{\mathrm{h}} / K_{2}=$ $c^{2} R_{\mathrm{w}} / 2 \pi G L\left(R_{\mathrm{w}}\right)$.

For small warps, we extend the trigonometric functions in Equation (12) around $\theta \sim 0$ and it is trivial to show that the corresponding solution decays exponentially as

$$
\theta(t)=\theta_{0} \exp \left(-\frac{t}{T}\right) .
$$

This is the analytic solution found in Scheuer \& Feiler (1996, see their Equation (11)) ${ }^{3}$ and Martin et al. (2007, see their Equation (52)) and the time scale of the exponential decay $T$ exactly agrees with their derivations.

\subsubsection{Phase II: Gas Fueling Quenched}

After gas fueling is quenched, i.e., $\dot{J}_{\mathrm{f}}=0$, the total angular momentum of the system (black hole and accretion disk) becomes conserved. The disk subjects to both alignment and viscous diffusion. The former decreases the angular momentum of the disk $\left(K_{2}>0\right.$ in Equation (9)), whereas the later transfers the angular momentum outwards. Substituting Equations (8) and (9) into Equation (10) and with some simple mathematical implementations, we arrive at

$$
\frac{d \cos \theta}{d t}=-\frac{\dot{M}_{\mathrm{h}} \ell_{\mathrm{ms}}}{J_{\mathrm{d}}} \sin ^{2} \theta+\frac{K_{2}}{J_{\mathrm{h}}} \sin ^{2} \theta\left(1+\frac{J_{\mathrm{h}}}{J_{\mathrm{d}}} \cos \theta\right) .
$$

Following Kumar et al. (2008), we employ an approximate global model to simulate the influence of mass accretion on the disk properties so that accretion rate $\dot{M}$ can be determined in a quite simple fashion. To this end, we define the effective radius of the disk $R_{\mathrm{d}}$ as

$$
\frac{J_{\mathrm{d}}}{M_{\mathrm{d}}}=\ell\left(R_{\mathrm{d}}\right)=\left(G M_{\mathrm{h}} R_{\mathrm{d}}\right)^{1 / 2} .
$$

The mean global accretion rate is then given by

$$
\dot{M}_{\mathrm{h}}=-\frac{d M_{\mathrm{d}}}{d t}=\frac{M_{\mathrm{d}}}{t_{\mathrm{acc}}},
$$

where the accretion time scale reads

$$
t_{\text {acc }} \sim \frac{R_{\mathrm{d}}^{2}}{\nu_{1}\left(R_{\mathrm{d}}\right)},
$$

where $\nu_{1}$ is the normal viscosity associated to the azimuthal shear due to the differential rotation of the disk. With the standard $\alpha$-prescription, $\nu_{1}$ is expressed by

$$
\nu_{1}=\alpha \Omega_{\mathrm{K}} H^{2}=\alpha h^{2} \Omega_{\mathrm{K}} R^{2},
$$

where $h=H / R$ is the disk's aspect ratio and $\Omega_{\mathrm{K}}$ is the Keplerian angular momentum. Combining above equations, we can obtain

$$
\dot{M}_{\mathrm{h}}=\frac{\alpha h^{2} G^{2} M_{\mathrm{h}}^{2} M_{\mathrm{d}}^{4}}{J_{\mathrm{d}}^{3}} .
$$

This equation apparently indicates the influences of alignment and accretion on $\dot{M}_{\mathrm{h}}$. From Equation (9), alignment processes reduce the disk angular momentum $J_{\mathrm{d}}$ at a rate of $\sim K_{2}$ but leave the disk mass $M_{\mathrm{d}}$ unchanged, therefore, increasing the

\footnotetext{
${ }^{3}$ Note that Scheuer \& Feiler (1996) omitted a factor of $\sqrt{2}$ in their Equation (11).

${ }^{4}$ We stress that the definition of $R_{\mathrm{d}}$ aims to simulate the disk evolution in a global way. It has the same order of magnitude as, but is not necessarily identical to, the disk's physical outer truncated radius.
} 

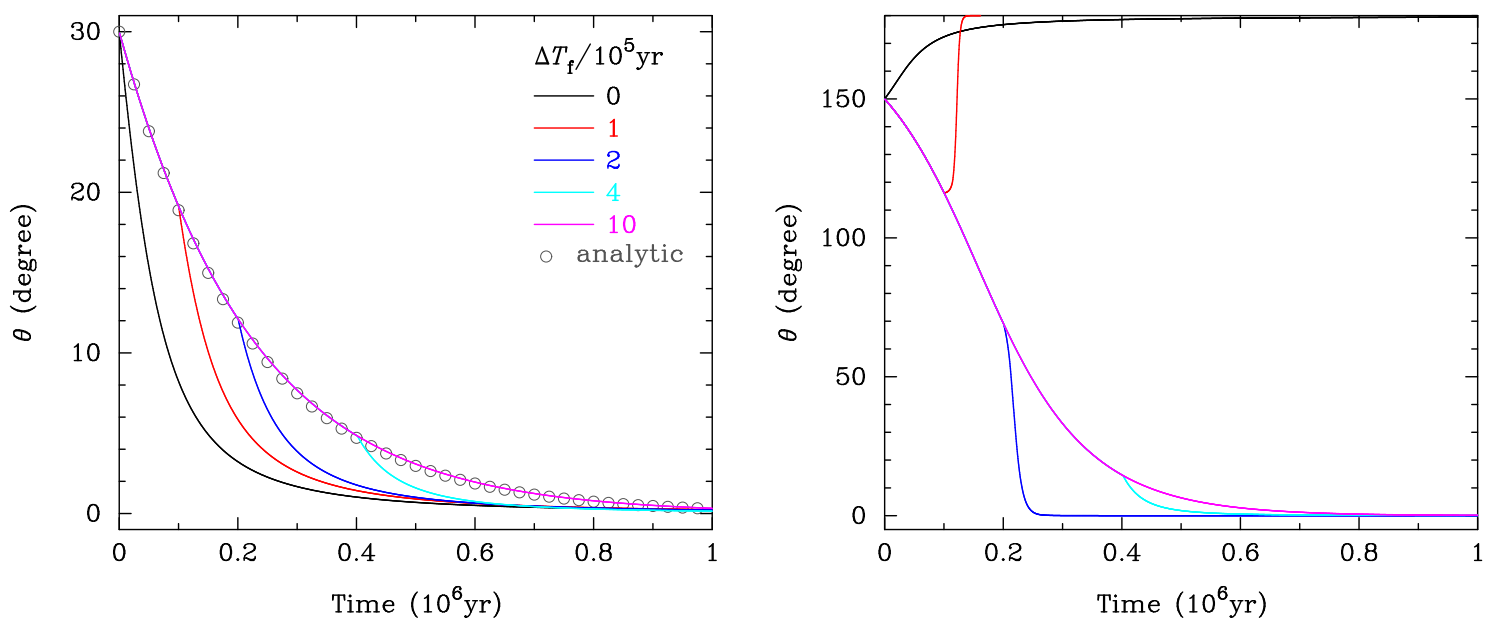

FIG. 1.- Evolution of inclination angle $\theta$ between black holes and disks for different lifetimes of gas fueling $\Delta T_{\mathrm{f}}$. The initial values are (Left) $\theta=30^{\circ}$ and (Right) $\theta=150^{\circ}$, respectively. Open circles in left panel represent the analytic solution for small warps: an exponentially decay with an e-folding timescale $T=2.2 \times 10^{5} \mathrm{yr}$ (see Equation 13 ).
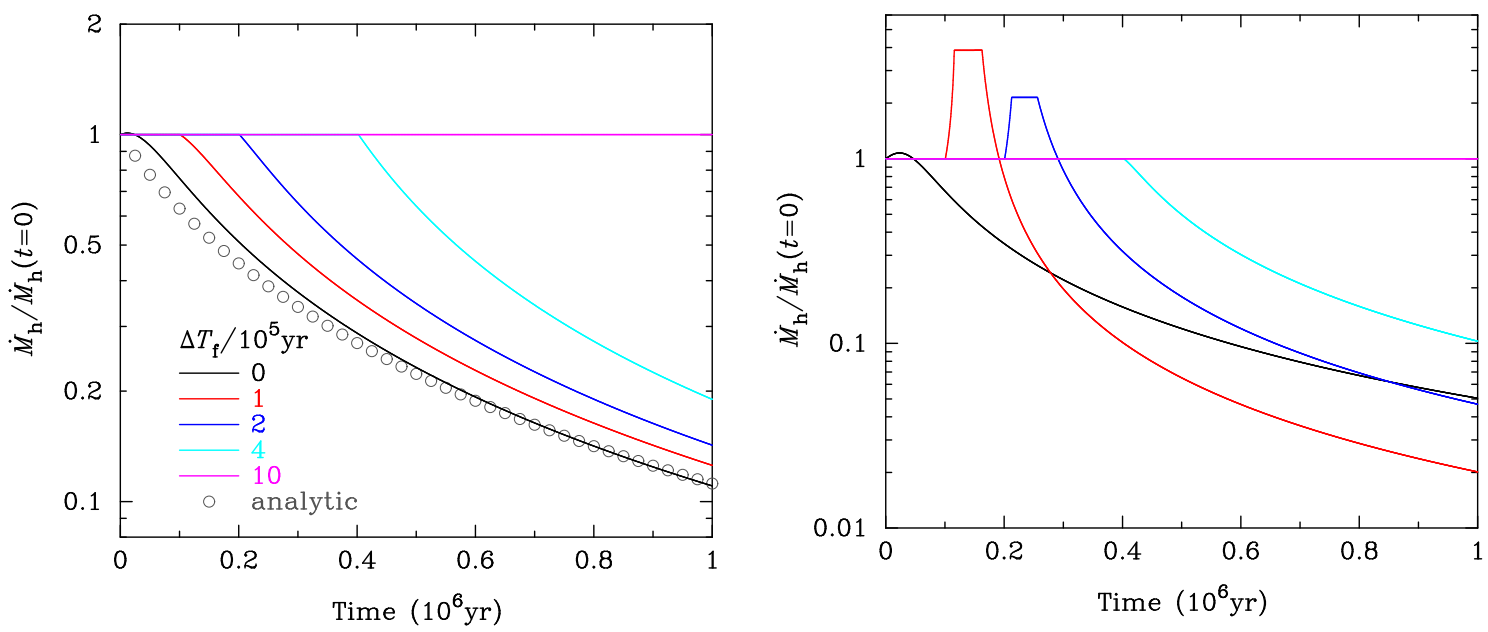

FIG. 2.- Same as Figure 1 but for mass accretion rate. The initial mass accretion rate is $\dot{M}_{\mathrm{h}}(t=0)=0.25 \dot{M}_{\text {Edd }}$. In left panel, open circles represent the asymptotic evolution following Equation [21. In right panel, mass accretion rates are limited by the Eddington rate.

accretion rate $\dot{M}_{\mathrm{h}}$. On the contrary, mass accretion reduces the disk mass but approximately keeps the disk angular momentum unchanged, considering that angular momentum loss due to the accretion inflow through the marginal stable orbit is usually negligible, therefore decreasing $\dot{M}_{\mathrm{h}}$.

Indeed, if only considering the influence of accretion rate, there exists an asymptotic solution to Equation (16) (see Kumar et al. 2008)

$$
M_{\mathrm{d}}(t)=M_{\mathrm{d}}(t=0)\left[1+\frac{3 t}{t_{\mathrm{acc}}^{\prime}}\right]^{-1 / 3},
$$

and

$$
\dot{M}_{\mathrm{h}}(t)=\frac{M_{\mathrm{d}}(t=0)}{t_{\mathrm{acc}}^{\prime}}\left[1+\frac{3 t}{t_{\mathrm{acc}}^{\prime}}\right]^{-4 / 3},
$$

where $t_{\mathrm{acc}}^{\prime}=t_{\mathrm{acc}}(0)$ is the accretion time scale at $t=0$. For small warp cases, we expect that the mass accretion rate approaches the above asymptotic behaviors, i.e., $\dot{M}_{\mathrm{h}} \propto t^{-4 / 3}$ for $t \gg t_{\text {acc }}^{\prime}$.

With mass accretion rate, we now rewrite $K_{2}$ in a more convenient form. Previous studies showed that the vertical viscosity $\nu_{2}$ is related to the normal azimuthal viscosity $\nu_{1}$ by
Ogilvie 1999; Lodato \& Pringle 2007; see also Perego et al. 2009)

$$
\frac{\nu_{2}}{\nu_{1}}=\frac{f_{\nu}}{2 \alpha^{2}},
$$

where $f_{\nu}$ is a coefficient of order of unity. As a zeroth-order approximation, we neglect the detailed shape of warped accretion disks and make use of the estimate for surface density $\Sigma \approx M_{\mathrm{h}} / 3 \pi \nu_{1}$ and for the angular momentum $L(R) \approx$ $\dot{M}_{\mathrm{h}} \sqrt{G M_{\mathrm{h}} R} / 3 \pi \nu_{1}$ as in flat disks. This is reasonable since accretion disks reach maximally warped around the warp radius $R_{\mathrm{W}}$ and then rapidly approach flat geometry beyond $R_{\mathrm{W}}$ (Scheuer \& Feiler 1996; Martin et al. 2007; Chen et al. 2009; Li et al. 2013). Combining Equations (2) and (7) gives

$$
K_{2} \approx \frac{f_{\nu} \dot{M}_{\mathrm{h}}}{6 \alpha^{2}} \sqrt{G M_{\mathrm{h}} R_{\mathrm{w}}} .
$$

For small warp amplitude, $f_{\nu}=1$, whereas for large warp amplitude, $f_{\nu}$ moderately decreases below unity, depending on the warp amplitude (Ogilvie 1999; Lodato \& Price 2010). Ogilvie (1999) developed a prescription to determine $f_{\nu}$ for any warp amplitude. However, this needs to know the detailed warp profile as a function of radius, therefore beyond 
LI et al.
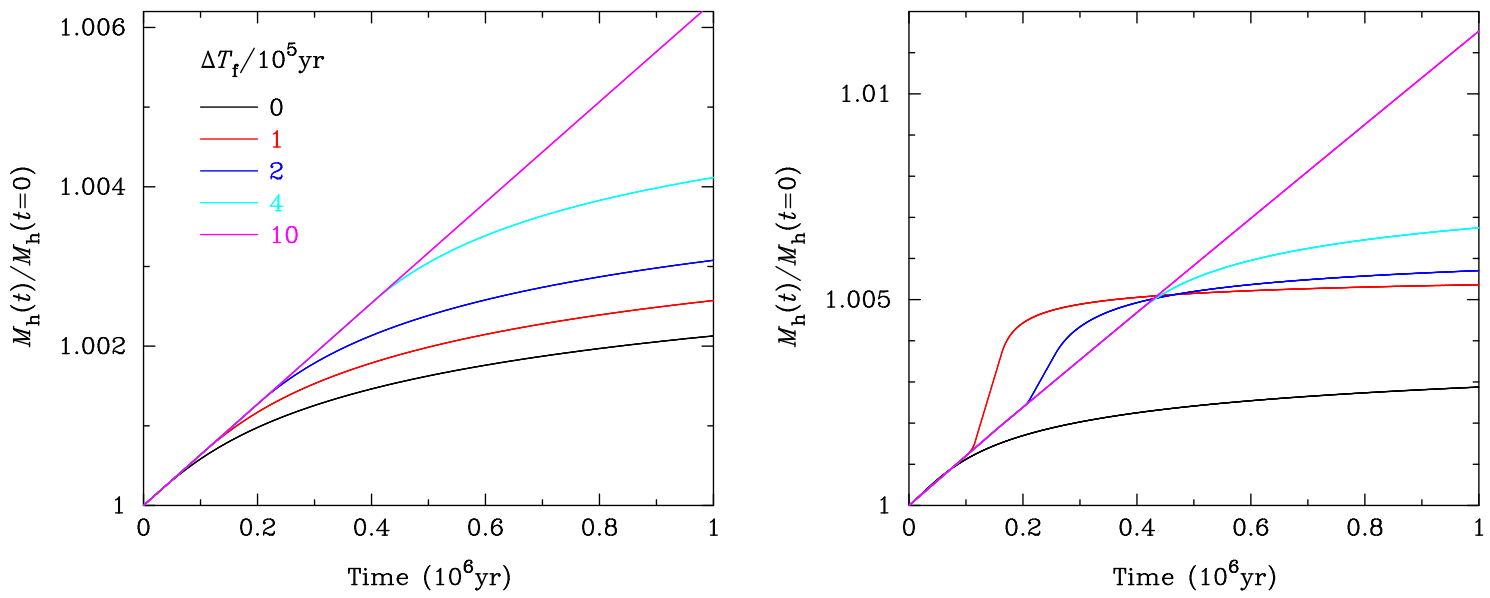

FIG. 3. - Same as Figure 1 but for mass growth of black holes. The initial mass of the black hole is $M_{\mathrm{h}}(t=0)=10^{8} M_{\odot}$.
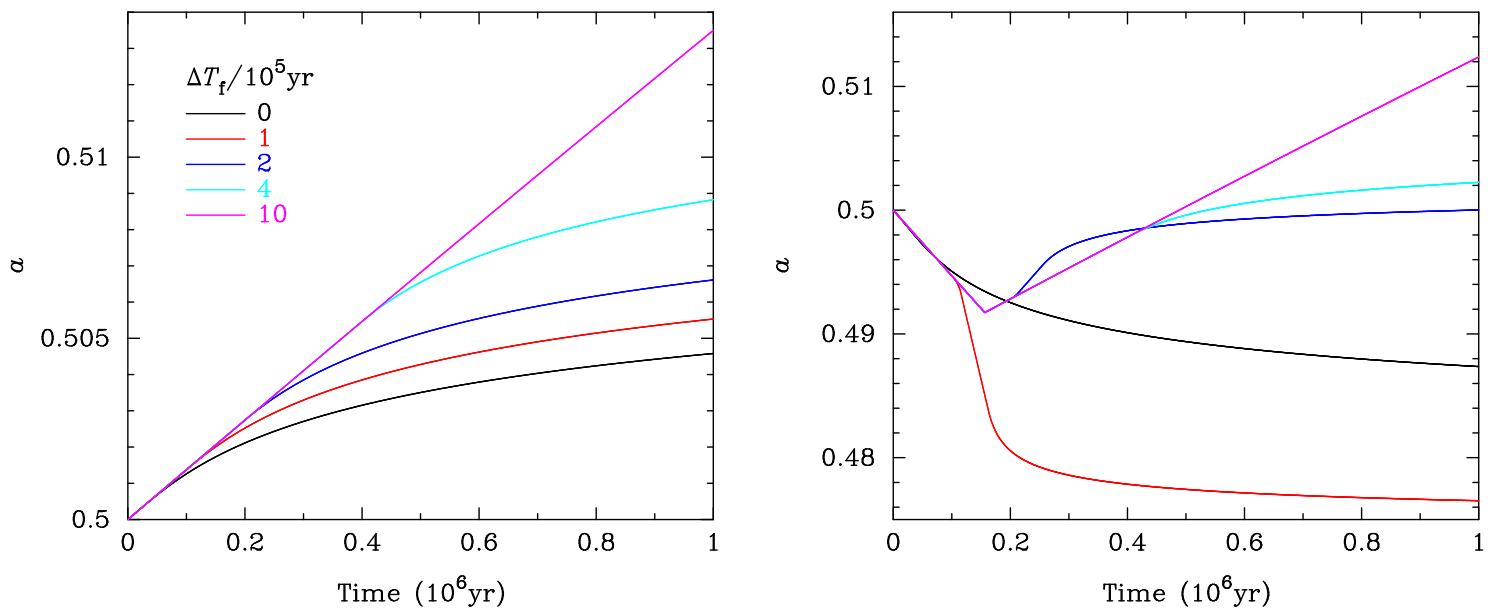

FIG. 4. - Same as Figure 1 but for spin evolution of black holes. The initial black hole spin is $a=0.5$.

the scope of the present work. We simply set $f_{\nu}=1$ throughout our calculations and below we will show the reliability of our results in light of this simplification.

\subsection{Black Hole Evolution}

The direction of the inner flat region of the disk depends on the inclinations of the incoming gas flow and the black hole: if $\theta \leq \pi / 2$, it aligns with the hole; while if $\theta>\pi / 2$, it anti-aligns with the hole. By taking into account the mass loss carried away by radiation, one obtains that the black hole grows as

$$
\frac{d M_{\mathrm{h}}}{d t}=(1-\eta) \dot{M}_{\mathrm{h}},
$$

and by combining with Equation (8), the black hole spin evolves as

$$
\frac{d a}{d t}=(1-\eta) \frac{\dot{M}_{\mathrm{h}}}{M_{\mathrm{h}}}\left(\frac{1}{1-\eta} \frac{\ell_{\mathrm{ms}}}{R_{\mathrm{g}} c}-2 a\right)
$$

where $\eta$ is the radiative efficiency depending on the spin parameter $a$ via

$$
\eta=1-\sqrt{1-\frac{2}{3 r}}
$$

and the specific angular momentum is calculated as

$$
\ell_{\mathrm{ms}}= \pm \sqrt{G M_{\mathrm{h}} R_{\mathrm{ms}}} \frac{1 \mp 2 a r^{-3 / 2}+a^{2} r^{-2}}{\left(1-3 r^{-1} \pm 2 a r^{-3 / 2}\right)^{1 / 2}}
$$

for $a<1$ and

$$
\ell_{\mathrm{ms}}= \pm \sqrt{G M_{\mathrm{h}} R_{\mathrm{ms}}} \frac{1 \pm r^{-1 / 2}+r^{-1} \mp r^{-3 / 2}}{\left(1 \pm 2 r^{-1 / 2}\right)^{1 / 2}}
$$

for $a=1$, where $r=R_{\mathrm{ms}} / R_{\mathrm{g}}, R_{\mathrm{g}}=G M_{\mathrm{h}} / c^{2}$ is the gravitational radius, and the upper sign refers to prograde accretion while the lower sign refers to retrograde accretion (Bardeen et al. 1972)

\section{RESULTS}

According to the standard accretion disk model, the disk's aspect ratio $h=H / R$ is in an order of $10^{-3} \sim 10^{-2}$ and weakly depends on radius in proportional to $R^{-1 / 20}$, on black hole mass in proportional to $M_{\mathrm{h}}^{-1 / 10}$, and on mass accretion rate in proportional to $\dot{M}_{h}^{1 / 5}$ (Shakura \& Sunvaev 1973; see also Collin-Souffrin \& Dumont 1990; Natarajan \& Pringle 1998; King et al. 2008). An accretion disk becomes self-gravitating when its mass reaches a factor $h$ of the black hole mass

${ }^{5}$ Note that, according to the calculation of Thorne (1974), the radiation emitted by the geometrically-thin disk and swallowed by the hole produces a counteracting torque that prevents the hole span up beyond an upper limit of $a \approx 0.998$. In our calculations, the accretion may proceed from retrograde to prograde fashion over episodes. This effectively means than the black hole spin may suffer a flip from $a=-1$ to $a=1$. For the sake of simplicity, we neglect the counteracting torque and assume that black holes can carry any spin between -1 and 1 . 
(e.g., Pringle 1981). We therefore adopt the initial disk mass $M_{\mathrm{d}} \approx h M_{\mathrm{h}}$. The initial effective radius of the disk $R_{\mathrm{d}}$ is determined by the required initial mass accretion rate and accretion time scale given with $h$. To be specific, from Equation (19), $\dot{M}_{\mathrm{h}}$ can be alternatively expressed through $R_{\mathrm{d}}$ and $h$ by

$$
\begin{aligned}
\frac{\dot{M}_{\mathrm{h}}}{\dot{M}_{\text {Edd }}}= & 0.25\left(\frac{\alpha}{0.1}\right)\left(\frac{\eta}{0.1}\right)\left(\frac{h}{5 \times 10^{-3}}\right)^{3} \\
& \times\left(\frac{R_{\mathrm{d}}}{2.7 \times 10^{3} R_{\mathrm{g}}}\right)^{-3 / 2}\left(\frac{M_{\mathrm{h}}}{10^{8} M_{\odot}}\right)^{-1} .
\end{aligned}
$$

The corresponding accretion time scale is

$$
\begin{aligned}
t_{\mathrm{acc}}= & 8.8 \times 10^{5} \mathrm{yr}\left(\frac{\alpha}{0.1}\right)^{-1}\left(\frac{h}{5 \times 10^{-3}}\right)^{-2} \\
& \times\left(\frac{R_{\mathrm{d}}}{2.7 \times 10^{3} R_{\mathrm{g}}}\right)^{3 / 2}\left(\frac{M_{\mathrm{h}}}{10^{8} M_{\odot}}\right) .
\end{aligned}
$$

This is the typical timescale for accretion disks truncated by self-gravitational collapse (see, e.g., Perego et al. 2009). The ratio between the angular momenta of the disk and the hole is

$$
\frac{J_{\mathrm{d}}}{J_{\mathrm{h}}}=0.26 a^{-1}\left(\frac{h}{5 \times 10^{-3}}\right)\left(\frac{R_{\mathrm{d}}}{2.7 \times 10^{3} R_{\mathrm{g}}}\right)^{1 / 2} \text {. }
$$

Our calculating procedure is as follow. We start with a black hole that has a mass of $M_{\mathrm{h}}$ and a spin of $a$. The accretion disk is assigned an initial mass of $M_{\mathrm{d}}=h M_{\mathrm{h}}$ and angular momentum of $J_{\mathrm{d}}=M_{\mathrm{d}}\left(G M_{\mathrm{h}} R_{\mathrm{d}}\right)^{1 / 2}$. The initial inclination angle between the black hole hole and the disk is $\theta$. We then evolve Equations (9) and (16) for the angular momentum and mass of the disk, Equations (24) and (25) for the mass and spin of the black hole, and finally Equations (11) and (14) for the inclination angle. The mass accretion rate $\dot{M}_{\mathrm{h}}$ is determined by Equation (19). We keep in mind that in the phase with gas fueling, the disk's mass and angular momentum are unchanged. For a series of accretion events, we just repeat the above procedure but with the mass and spin of the black hole inherit from the previous episode.

We assume that in the beginning of an episode, the disk has already been formed and neglect the stage for the formation of the disk. It is expected that during the formation stage, the accretion rate must be very insignificant so that the influence on black hole spin evolution is negligible. Also, we implicitly assumed that the time lag between the successive episodes is long enough to let the disk be consumed on the accretion time scale before the next episode starts. This can be verified by the fact that the observed "duty cycle" is generally much less than unity (e.g., Shankar et al. 2009; Li et al. 2012). Duty cycle measures the fraction of active black holes to total black holes, and equivalently indicates the time fraction of active phases over total lifetime (active and inactive) for a single black hole (Wang et al. 2006).

In following calculations, unless stated otherwise, we set $\alpha=0.1, h=5 \times 10^{-3}, R_{\mathrm{d}}=2.7 \times 10^{3} M_{8}^{-2 / 3} \eta_{-1}^{2 / 3} R_{\mathrm{g}}, M_{\mathrm{h}}=$ $10^{8} M_{\odot}$, and $a=0.5$ as the fiducial values. It is worth mentioning that the choice of $R_{\mathrm{d}}$ dependent on black hole mass and the radiative efficiency aims to let the dimensionless accretion rate $\dot{M}_{\mathrm{h}} / \dot{M}_{\text {Edd }}$ at about 0.25 , the typical value observed in luminous AGN surveys (Kollmeier et al. 2006; Shen et al. 2008; Li et al. 2011). We also set an upper limit of mass accretion rate by $\dot{M}_{\mathrm{h}} \leq \dot{M}_{\mathrm{Edd}}$ considering that beyond the Ed- dington limit, radiative feedback may be important to regulate the accretion rate at around the Eddington limit.

\subsection{Alignments and Anti-Alignments}

In Figure 11 we demonstrate evolution of the inclination angle between the disk and the black hole for different lifetimes of gas fueling: $\Delta T_{\mathrm{f}}=(0,1,2,4,10) \times 10^{5} \mathrm{yr}$. The initial value of $\theta$ is set to be $\theta_{0}=\pi / 6$ and $\theta_{0}=5 \pi / 6$ in the left and right panels, respectively. As can be seen, for the case of $\theta_{0}=\pi / 6$, the inclination angle rapidly decreases on a timescale of $10^{5} \mathrm{yr}$, which is the typical estimate in previous numerous literature ( see, e.g., Perego et al. 2009; Li et al. 2013 and references therein). As the lifetime of gas fueling $\Delta T_{\mathrm{f}}$ increases, the decay rate of $\theta$ is slightly reduced. This is because during the gas fueling, the disk maintains its orientation and only the black hole is driven to align to the disk. While after the gas fueling is quenched, both the disk and the hole change their orientations towards the direction of the total angular momentum of the system. This can be further verified from Equations (11) and (14). Interestingly, the behavior of $\theta$ for $\Delta T_{\mathrm{f}}=10^{6} \mathrm{yr}$, namely, the disk is continuously fed throughout the time range of calculations, is exactly identical to the analytic solution found in Scheuer \& Feiler (1996) and Martin et al. (2007).

The evolution of $\theta$ is much more complicated for the case of $\theta_{0}=5 \pi / 6$ as shown in the right panel of Figure 1 With no gas fueling $\left(\Delta T_{\mathrm{f}}=0\right)$, the inclination angle rises up to $\pi$ all the way. Indeed, the instantaneous behavior of $\theta$ is governed by the ratio $J_{\mathrm{d}} / J_{\mathrm{h}}$ in Equation (14): for $J_{\mathrm{d}} / J_{\mathrm{h}}>-\cos \theta$, the inclination $\theta$ decreases, while for $J_{\mathrm{d}} / J_{\mathrm{h}}<-\cos \theta$, the inclination $\theta$ increases (see also Li et al. 2013). As $\Delta T_{\mathrm{f}}$ increases, the final configuration of the system transits from anti-alignment $(\theta=\pi)$ to co-alignment $(\theta=0)$. We note there is a steep decreasing/increasing trend of $\theta$ just after the gas fueling is quenched, ascribed to the rapid reduce of the angular momentum of the disk and hence enhancement of mass accretion rate due to the viscous dissipation arising from alignments. See below for detailed explanations.

Figure 2 shows the corresponding mass accretion rates. For the sake of comparison, the asymptotic evolution of accretion rate by Equation (21) is superposed in the left panel. If there is no gas fueling, as the inclination angle tends to zero, the angular momentum of the disk approach constant. As a result, the mass accretion rate evolves asymptotically following Equation (21). In the right panel, the initial value of $\theta$ is $\theta_{0}=5 \pi / 6$. Instead of a gradual decay, there exists a notable peak of accretion rate, which even reaches the Eddington limit $\dot{M}_{\text {Edd }}$ in some cases. The similar behavior of an enhancement of the mass accretion rate also appears in the previous numerical simulations on warped accretion disks (see, e.g., Lodato \& Pringle 2006 and Nixon et al. 2012). This can be easily understood in terms of Equations (9), (15), and (16): strong dissipation arising from alignments leads to an intense reduce of the magnitude of $J_{\mathrm{d}}$; the effective radius $R_{\mathrm{d}}$ accordingly shrinks to maintain a Keplerian rotating disk. As a consequence, the accretion time scale $t_{\text {acc }}$ is shortened and mass accretion rate $\dot{M}_{\mathrm{h}}$ is significantly enhanced. In reality, emergence of the enhancement of accretion rate depends on competition between the viscous-dissipation driven deduce of $J_{\mathrm{d}}$ (due to warp alignments) and mass-accretion driven deduce of $M_{\mathrm{d}}$. This is the reason why there is no accretion rate enhancement for some curves in Figure 2 .

By assuming the angular momentum conservation of the 


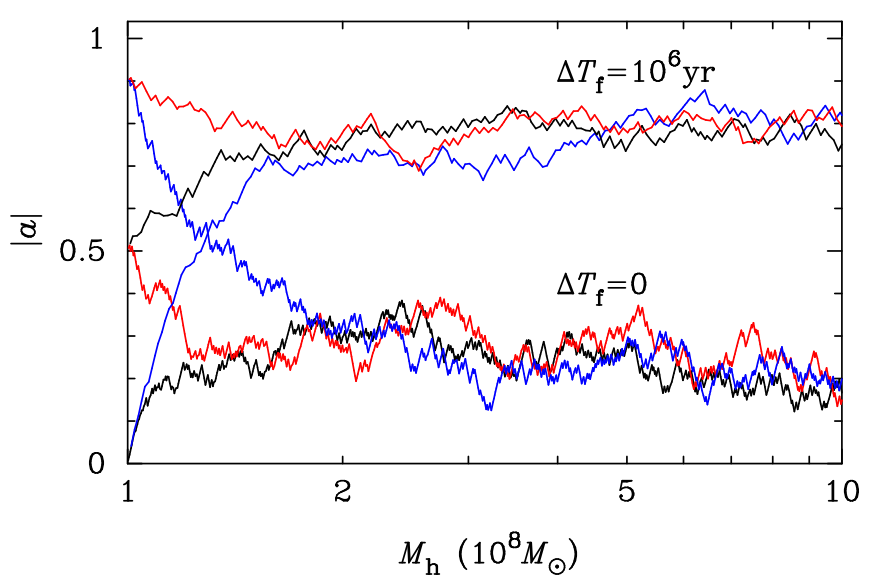

FIG. 5.- Evolution of spin magnitude with mass growth during a series of accretion events. The initial spin is $a=0.9,0.5$, and 0.0 for red, blue, and black lines, respectively. The gas fueling lifetime is $\Delta T_{\mathrm{f}}=10^{6} \mathrm{yr}$ for upper three lines, and there is no gas fueling for lower three lines.

system, King et al. (2005) derived a condition for occurrence of anti-alignments: the initial angle between the disk and the hole satisfies $\cos \theta_{0}<-J_{\mathrm{d}} / 2 J_{\mathrm{h}}$. This equation is intensively used in subsequent studies on cosmological evolution of SMBH spins (e.g., Volonteri et al. 2007; King et al. 2008; Lagos et al. 2009; Fanidakis et al. 2011; Dotti et al. 2013; Dubois et al. 2014; (Sesana et al. 2014). We relax the assumption of the conservation of angular momentum and let the accretion disk steadily fueled for a lifetime of $\Delta T_{\mathrm{f}}$. Since the total angular momentum of the system is not conserved, it is inappropriate to directly use the King et al. (2005)'s condition. In the right panel of Figure 1 the initial ratios $J_{\mathrm{d}} / J_{\mathrm{h}}=0.40$ and $\cos \theta_{0}=-0.87$, therefore the condition $\cos \theta_{0}<-J_{\mathrm{d}} / 2 J_{\mathrm{h}}$ is well established. However, for $\Delta T_{\mathrm{f}} \gtrsim 2 \times 10^{5} \mathrm{yr}$, the system ends up an alignment instead of an anti-alignment.

Nevertheless, the ratio $J_{\mathrm{d}} / J_{\mathrm{h}}$ remains an important indicator of alignments or anti-alignments (if there is no gas fueling). Small ratios mean that the angular momentum of black holes dominates over that of disks, therefore it is comparatively easier to drive the disks towards alignments or anti-alignments. Conversely, if the angular momentum of the disks is dominated, the holes are "lighter" to change their orientations. This is somehow similar to the cases that the disks are continuously fueled: the holes tend to align towards the disks (see also the discussions of King et al. 2005 and Lodato \& Pringle 2006).

\subsection{Spin Evolution}

\subsubsection{A Single Accretion Event}

In Figures 3 and 4 we show evolution of the black hole's properties, i.e., mass and spin, respectively, for initial inclination angles $\theta_{0}=\pi / 6$ and $\theta_{0}=5 \pi / 6$ with a set of gas fueling lifetimes $\Delta T_{\mathrm{f}}$. Compared with the case of $\theta_{0}=\pi / 6$, we can find that the black hole mass growth for $\theta_{0}=5 \pi / 6$ is much more efficient. This is because the Eddington limit is larger with lower radiative efficiency due to retrograde accretion for $\theta>\pi / 2$ and also because the accretion rate is enhanced as plotted in Figure 2 The spin evolution in Figure 4 clearly illustrates the important influences of gas fueling. As the lifetime of gas fueling increases, the black hole is gradually driven to align with the disk regardless of the initial inclination. As a result, in right panel of Figure 4 there exists a trend for $\Delta T_{\mathrm{f}} \gtrsim 2 \times 10^{5} \mathrm{yr}$ that spin first declines due to retrograde accretion and then increases when the inclination angle transits to $\theta<\pi / 2$. In addition, it can be found that the spin changes are relatively more significant for black holes with $\Delta T_{\mathrm{f}}<2 \times 10^{5} \mathrm{yr}$, because these holes undergo retrograde accretion throughout the episode, which carries larger angular momentum compared with the prograde accretion (see Equations (27) and (28).

\subsubsection{A Series of Accretion Events}

The spin of black holes will reach an equilibrium value after a series of accretion events, depending on the fraction of prograde and retrograde accretion (King et al. 2008; Sesana et al. 2014). Evidently, this fraction is intimately relevant to the degrees of anisotropy in the orientations of accretion disks with respect to the holes. If all the accretion events occur with the initial inclination angles $\theta_{0}<\pi / 2$, the black holes accrete in a coherent fashion and their spin will be spun up all the way to the maximum $(a=1)$. On the other hand, the inclination angles $\theta_{0}$ may distribute randomly over the whole space $(0 \sim \pi)$ (e.g., King et al. 2008; Wang et al. 2009; Hopkins et al. 2012; Li et al. 2012). For simplicity, we only focus on the random distribution of $\theta_{0}$ in proportional to $\sin \theta_{0}$ over $(0 \sim \pi)$, so that both alignments and anti-alignments can occurs in our calculations. We first generate a random inclination angle and then apply the prescription described above to track down the mass growth and spin evolution of black holes. The calculation ends when the accretion rate decreases to one tenth of its initial value, i.e., $0.025 \dot{M}_{\text {Edd }}$.

In Figure 5, we illustrate how black hole spin evolves along their mass growth during a series of accretion events. We stress that we adopt the effective radius of accretion disks dependent on black hole mass as $R_{\mathrm{d}}=2.7 \times 10^{3} M_{8}^{-2 / 3} \eta_{-1}^{2 / 3} R_{\mathrm{g}}$ in order to guarantee the initial dimensionless accretion rate at 0.25 in Equation 29). Once there is no gas fueling, irrespective of the initial spin values, black holes lose the memory of their initial status and approach a relatively low spin roughly after doubling their mass. Then as mass growth, there is a weak trend that their spin gradually falls off. Let's give an intuitive explanation for such weakly negative dependence of spin on mass through the ratio $J_{\mathrm{d}} / J_{\mathrm{h}}=1$. Since we adopt $R_{\mathrm{d}} \propto M_{\mathrm{h}}^{-2 / 3}$, Equation (31) yields $a \propto M_{\mathrm{h}}^{-1 / 3}$. Of course, the real situation is much more complicated, but the negative dependence is retained (see also below for further discussions).

Once the disks are fueled for a lifetime longer that the typical alignment timescale (e.g., $\Delta T_{\mathrm{f}}=10^{6} \mathrm{yr}$ ), as expected, the holes are always aligned to the disks and are rapidly spun up to fast rotating. However, we note that the final spin never reaches the maximal value $a=1$ because during the alignments, there exists a period with retrograde accretion that spins down the holes. This indicates that even though the final configuration in an accretion event is alignment instead of anti-alignment, the spin decrease during the time with retrograde accretion still contributes to the final spin status and therefore should be appropriately included.

\section{IMPLICATIONS FOR SPIN DISTRIBUTION AND EPISODIC LIFETIMES OF AGNS}

\subsection{Monte-Carlo Simulations}

We perform Monte-Carlo simulations to explore the spin distribution with the above described prescription. The free parameters $\alpha, h$, and $R_{\mathrm{d}}$ are set by their fiducial values as in Section 4. Initially, we assign to a black hole a mass drawn from a uniform distribution in logarithm over $\left(10^{6} \sim 10^{8}\right) M_{\odot}$ 

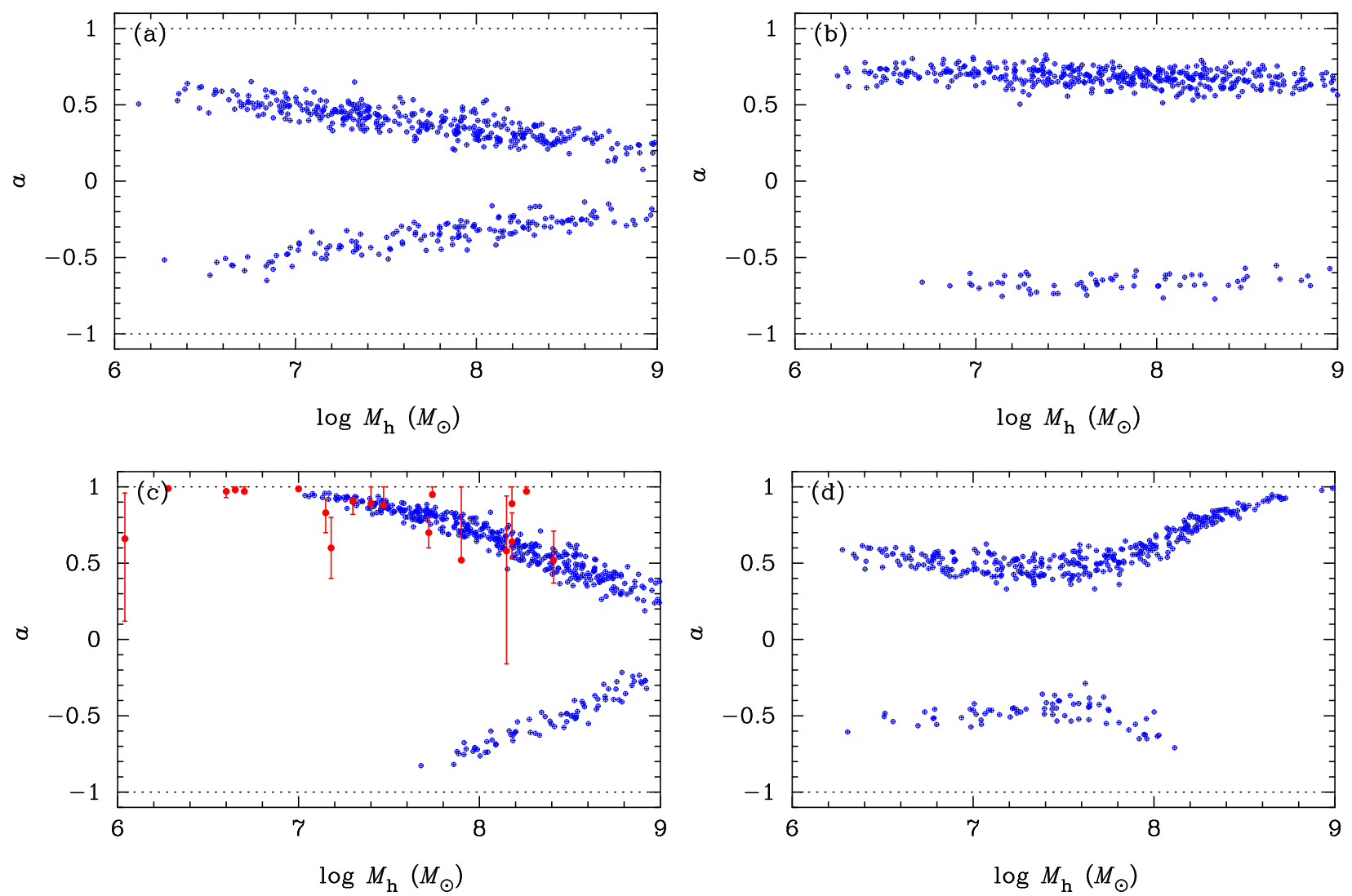

FIG. 6.- Spin distribution with black hole mass from Monte-Carlo simulations for different gas fueling lifetime $\Delta T_{\mathrm{f}}$ : (a) 0 , (b) $10^{5} \mathrm{yr}$, (c) $10^{5} M_{8}^{-1} \mathrm{yr}$, and (d) $10^{5} M_{8}^{1.5} \mathrm{yr}$. Negative spin means that black holes are undergoing retrograde accretion. Data points with error bars in panel (c) are the measured spin through broad Iron K $\alpha$ line compiled by Brenneman (2013) and Revnolds (2013) (see also Table 2 of Sesana et al. 2014). Horizontal dotted lines represents black hole spin $a=1$ and -1 , respectively.

TABLE 1

EPISODIC LIFETIMES OF AGNS CONSTRAINED FROM OBSERVATIONS IN THE LITERATURE.

\begin{tabular}{ccccc}
\hline \hline Object & Redshift & Episodic Lifetime (Myr) & Telescope & Reference \\
\hline & \multicolumn{5}{c}{ Proximity Effect } \\
\hline Q 0302-003 & 3.285 & $10-30$ & VLT & 1,2 \\
HE 2347-4342 & 2.885 & $\sim 25$ & VLT & 3 \\
KP 76 & 2.466 & $\gtrsim 25$ & Keck, HIRES & 4 \\
KP 77 & 2.535 & $16-33$ & Keck, HIRES & 4 \\
HS 1700+6416 & 2.748 & $\gtrsim 20$ & HST/COS & 5 \\
Quasar Pair Sample & $\sim 2.2$ & $\sim 1$ & Keck, Lick, NOAO, SDSS & 6 \\
\hline & \multicolumn{5}{c}{ Kluorescent Ly $\alpha$ emission } & \\
\hline HS 1549+1919 & $\sim 2.84$ & $\gtrsim 1.3$ & VLT & 8 \\
QSO 0420-388 & $\sim 3.1$ & $\approx 60$ & Keck & 9 \\
Quasar Sample & $\sim 2.7$ & $1-20$ &
\end{tabular}

REFERENCES. - (1) Worseck \& Wisotzki (2006); (2) Jakobsen et al. [2003); (3) Worseck et al. (2007); (4) Gonçalves et al. (2008); (5) McQuinn \& Worseck (2014); (6) Kirkman \& Tytler (2008); (7) Adelberger et al. (2006); (8) Cantalupo et al. (2007); (9) Trainor \& Steidel (2013).

and a spin drawn uniformly over $(0 \sim 1)$. For this newly generated black hole, we first let it grow with 100 accretion episodes, to remove the memory of its initial conditions. Afterwards, this hole continues to accrete for a series of episodes. We randomly assign the number of episodes between $(0 \sim 900)$ so that in average the hole undergoes 500 times activities. We then record the final mass and spin information of the hole. In each accretion episode, the inclination $\theta_{0}$ between the hole and its accretion disk follows a uniform distribution of $\cos \theta_{0}$ over $(-1 \sim 1)$. An individual event is re- garded to be terminated once the accretion rate decreases below a tenth of its initial value. We repeat such procedure 500 times and are finally left with a sample of 500 black holes.

In Figure 6 we plot the spin distribution with black hole mass for different gas fueling lifetime $\Delta T_{\mathrm{f}}=0,10^{5} \mathrm{yr}$, $10^{5} M_{8}^{-1} \mathrm{yr}$, and $10^{5} M_{8}^{1.5} \mathrm{yr}$ in panels (a)-(d), respectively. In panel (a), there is no gas fueling and the disks will be consumed within an accretion timescale $t_{\mathrm{acc}} \sim 8.8 \times 10^{5} \mathrm{yr}$ (see Equation (30)). As the ratio $J_{\mathrm{d}} / J_{\mathrm{h}}$ decreases with black hole mass, the accretion disks of massive black holes are easier 


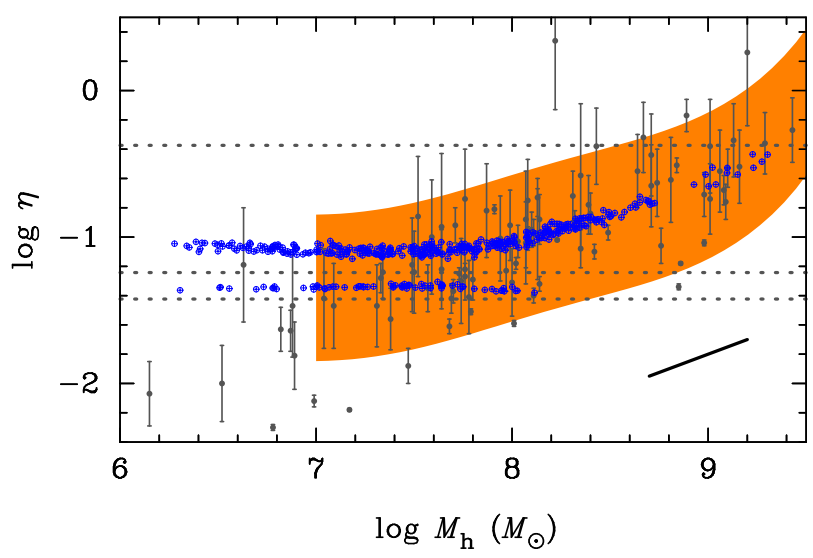

FIG. 7.- Radiative efficiency with black hole mass for gas fueling lifetime $\Delta T_{\mathrm{f}}=10^{5} M_{8}^{1.5} \mathrm{yr}$. Negative spin means that the black holes are undergoing retrograde accretion. Gray points with errorbars are the radiative efficiency of local quasars estimated by Davis \& Laor (2011) and shaded area represents the radiative efficiency at $z \sim 1$ estimated by Li et al. (2012). Horizontal dotted lines represents $\eta=0.42,0.057$, and 0.038 for black hole spin $a=1$, 0 , and -1 , respectively. To guide the eye, a solid line with a slope of 0.5 is plotted in the bottom right corner.

change their orientations and thus anti-alignments become more probable. This results in a negative correlation between spin and mass. A similar trend was also found by (Dotti et al. 2013, corresponding to $F=0$ in their model). When we switch on the gas fueling for a time of $\Delta T_{\mathrm{f}}=10^{5} \mathrm{yr}$ in panel (b), black holes are always driven to align towards the disks during this period. Therefore, the fraction of retrograde accretion is significantly reduced and all the black holes carry spin $|a| \gtrsim 0.5$.

We also choose a mass-dependent lifetime of gas fueling for the sake of comparison with observations. In panel (c), the gas fueling lifetime is $\Delta T_{\mathrm{f}}=10^{5} \mathrm{M}_{8}^{-1} \mathrm{yr}$, indicating that the disks surrounding $10^{7} M_{\odot}$ black holes will be fueled for $10^{6} \mathrm{yr}$, far longer than the alignment timescale. Accordingly, smaller black holes are overall spun up to maximum rotating. There is a strong anti-correlation between spin and mass. On the other hand, in panel (d), we reverse the mass dependence of fueling lifetime to $\Delta T_{\mathrm{f}}=10^{5} M_{8}^{1.5}$. As expected, massive black holes at $\sim 10^{9} M_{\odot}$ have maximum spin $(a=1)$ whereas lower massive black holes in this case are not affected (since $T_{\mathrm{f}}$ is much less that the accretion timescale and can be neglected) and just follow the distribution in panel (a).

\subsection{Comparison with Observations}

We now compare our calculated spin distributions with observations. Notwithstanding measuring spin of massive black holes is yet challenging so far, there are several developing techniques shedding useful light (e.g., Wang et al. 2009; Czerny et al. 2011; Davis \& Laor 2011; Li et al.|2012; Brenneman 2013; Done et al. 2013; Reynolds 2013; Wu et al. 2013). Among these techniques, we focus on the direct measurements using the broad Iron $\mathrm{K} \alpha$ line fitting (Brenneman 2013; Reynolds 2013 and references therein) and the indirect estimates using the radiative efficiency of thin accretion disks as a surrogate of the black hole spin (see Equation (26); Davis \& Laor 2011; Li et al. 2012). Both these two methods had been applied to a sample or a population of AGNs, allowing us to perform straightforward comparison. Unfortunately, each method bears a large source of uncertainties that are not well understood and the obtained results seem incompatible at this stage. Therefore, we perform comparison separately as follows.
In panel (c) of Figure 6 we superpose the spin of 19 sources measured from Iron K $\alpha$ lines, most of which are Seyfert I or narrow line Seyfert I AGNs residing in spirals or lenticular galaxies (see Sesana et al. 2014). Reynolds (2013) proposed that in this sample low massive black holes $\left(\sim 10^{\dagger} M_{\odot}\right)$ are spinning fast while black holes with mass $>10^{8} M_{\odot}$ may have moderate spin. Our calculations show that a lifetime of gas fueling as $\Delta T_{\mathrm{f}}=10^{5} \mathrm{M}_{8}^{-1}$ can generally reproduce such observed spin distribution. This means low massive black holes require longer gas fueling to reach alignments and to accrete coherently.

On the other hand, Davis \& Laor (2011) reconstructed the spectral energy distributions for a sample of 80 PalomarGreen quasars and estimated their individual radiative efficiency of accretion flows based on the thin disk model. They found a tight correlation between the efficiency with black hole mass approximately as $\eta \propto M_{\mathrm{h}}^{1 / 2}$ (but see also Raimundo et al.2012), plausibly implying a raise in the black hole spin with mass. Meanwhile, Li et al. (2012) applied the Soltan's argument (Sołtan 1982) to solve the continuity equation of SMBH demography in active and quiescent galaxies (Li et al. 2011) and quantified the radiative efficiency with redshift and black hole mass. Their results suggested that the efficiency undergoes cosmological evolution and generally there is an increasing trend of the efficiency with black hole mass at redshift $z \geq 1$ (see also Cao \& Li 2008; Volonteri et al. 2013; Ueda et al. 2014). Figure 7 compares the radiative efficiency from our calculations by adopting the gas fueling lifetime $\Delta T_{\mathrm{f}}=10^{5} M_{8}^{1.5} \mathrm{yr}$ (as in panel (d) of Figure 6) with these from observational constraints of Davis \& Laor (2011) and Li et al. (2012, at $z \sim 1$ ). Here, the radiative efficiency is calculated from black hole spin as Equation (26) by assuming that the thin disk model applies. As stated above, in the context of chaotic accretion without gas fueling, high massive black holes $\left(\sim 10^{9} M_{\odot}\right)$ have low spin because of net spindown by retrograde accretion with anti-alignments. Therefore, to maintain the observed high radiative efficiency (and high spin), sufficient prolonged gas fueling is in need for these holes.

Longer gas fueling means that there is plenty of gas reservoir progressively channeled into the accretion disks and the black holes are accordingly long-lived. There are several techniques developed for measuring or estimating episodic lifetimes of AGNs, see Martini (2004) for a review. In Table 1 we summarize the observational constraints on episodic lifetimes of AGNs through the proximity effect and the fluorescent Ly $\alpha$ emission. Note that both these constraints are presently only realizable for high-redshift, luminous quasars, indicating that there reside high massive black holes (plausibly $>10^{9} M_{\odot}$ ). It seems that the observed AGN episodic lifetimes are generally $\sim 10^{7} \mathrm{yr}$ but with large uncertainties. Such long lifetimes strongly imply that high massive black holes are rapidly spinning at high redshift, consistent with previous studies probed through the radiative efficiency (e.g., Wang et al. 2009; Li et al. 2012; Trakhtenbrot 2014). Our calculations in the present work further show that if the correlation between the efficiency and the black hole mass is reliable, low massive black hole must accrete in more chaotic way and a gas fueling lifetime as $\Delta T_{\mathrm{f}} \sim 10^{5} M_{8}^{1.5}$ can roughly reproduce the observed slope $\eta \propto M_{\mathrm{h}}^{1 / 2}$. This gives rise to an episodic lifetime of $>3 \times 10^{6} \mathrm{yr}$ for SMBHs with $M_{\mathrm{h}}>10^{9} M_{\odot}$, coincident with the observations. 


\section{DISCUSSIONS AND CONCLUSIONS}

The main differences of the present work compared with the previous studies (e.g., Perego et al. 2009; Dotti et al. 2013) lie at: 1) we include the influences of continuous gas fueling on the alignments between black holes and their accretion disks; and 2) we consider the finite-size accretion disks (see also King et al. 2008). There are several simplifications in our calculations meriting further explanations. First, we neglect the detailed structures and properties of accretion disks and employ an approximate global model to simulate the evolution of disks under alignments and accretion (Kumar et al. 2008). We are thus unable to handle the angular momentum transportation throughout the disks. This may be important when the disks suffer large warps $(\theta \sim \pi / 2)$ in which non-linear effects cause the disk structures around the warp radius to change dramatically and the simple global approximate plausibly becomes invalid. However, since the spin parameter invokes the whole accretion history, we expect that our results are insensitive to this effect. Secondly, the main free parameters in our calculations are the disk's aspect ratio $h$ and the effective radius $R_{\mathrm{d}}$. In terms of the mass accretion rate, $h$ and $R_{\mathrm{d}}$ is degenerated along lines of constant $h^{2} R_{\mathrm{d}}^{-1}$. As mentioned above, the standard accretion disk model shows that $h$ is almost insensitive to radius and only weakly depends on black hole mass and mass accretion rate (Natarajan \& Pringle 1998; King et al. 2008). We therefore fix $h$ to the intermediate value between $10^{-3} \sim 10^{-2}$. The effective radius is chosen by a specified mass accretion rate in Equation 29, which is roughly at $\sim 0.25 \dot{M}_{\text {Edd }}$ from AGN surveys. We recalculate the spin distribution for $h$ between $10^{-2}$ and $10^{-3}$ and find the results are qualitatively unchanged. Thirdly, the Lense-Thirring torque $K_{2}$ calculated by Equation (23) is also a well approximate for small warps (Lodato \& Pringle 2006). When disks are strongly warped, on one hand, the relation $\dot{M} \approx 3 \pi \nu_{1} \Sigma$ no longer applies, in particular in the region around the warp radius; on the other hand, the vertical shear viscosity $v_{2}$ deviates from the relation in Equation (22) and turns to be decreasing as warp amplitude increases (Ogilvie 1999; Lodato \& Price 2010). To see how our results depend on $f_{\nu}$, we artificially set an extreme value of $f_{\nu}=0.1$ for large inclination angle $\theta \in(\pi / 3-2 \pi / 3)$ and show the comparison with $f_{\nu}=1$ in Figure 8 . The choice of a such range is based on the study by Perego et al. (2009) who found that the non-linear term becomes important for angular momentum transportation when the inclination angle $>\pi / 3$. Black hole spin magnitude is systematically smaller because of the reduced torque $K_{2}$ for large warps. However, we can find that the changes are moderate with such an extreme $f_{\nu}$ value and the overall spin distribution with mass is preserved.

For simplicity, we also assume that disk fueling is chaotic so that the initial inclination angle $\theta_{0}$ between the disks and black holes distributes randomly over $(0 \sim \pi)$. Dotti et al. (2013) proposed a comprehensive modeling on the anisotropy of $\theta_{0}$ using a parameter $F$ to describe the fraction of accretion events with $\theta_{0}>\pi / 2$. Therefore, $F=0.5$ stands for fully chaotic fueling and $F=0$ stands for fully coherent accretion. Sesana et al. (2014) further extended this prescription and connected the anisotropy parameter $F$ with the dynamic properties of the host galaxies. They postulated that the ratio of the rotation velocity to the velocity dispersion of the galaxies mirrors the degree of anisotropy of the gas component channeled into the nuclear regions. They argued that the rotation velocity measures the bulk rotation of the galaxies while

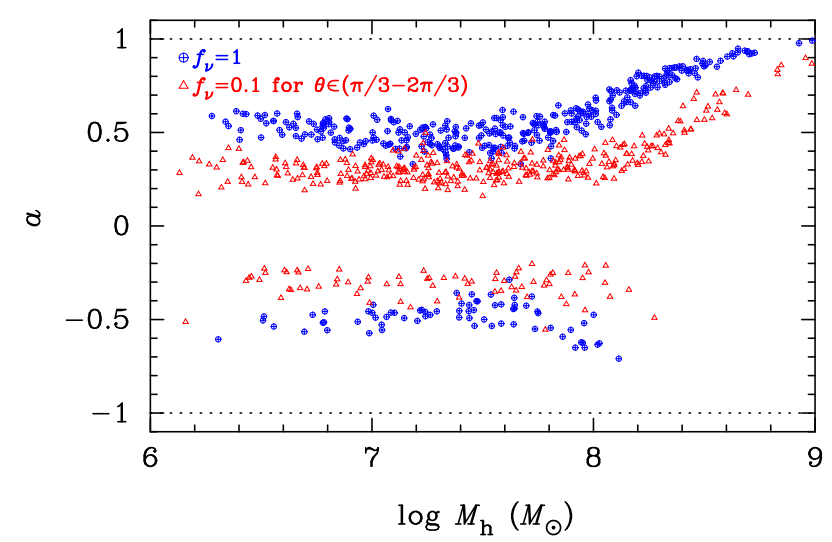

FIG. 8.- Influence of $f_{\nu}$ on spin distribution. Blue points represent the case $f_{\nu}=1$ for all the $\theta$ s and red points represent the case $f_{\nu}=0.1$ for $\theta \in$ $(\pi / 3-2 \pi / 3)$. The gas fueling lifetime is $\Delta T_{\mathrm{f}}=10^{5} M_{8}^{1.5} \mathrm{yr}$.

the velocity dispersion measures how chaotic the galaxies are. This seems in line with the recent finding of Ho \& Kim (2014) that the virial factor in reverberation mapping, which encodes the geometric and kinetic properties of the broad-line regions of AGNs, is related with the large-scale morphology of the host galaxy bulges. However, numerical simulations showed that the angular momentum of gas inflowing into the nuclear regions $(\sim \mathrm{pc})$ will lose its memory of the initial direction on the the larger scales ( $\sim \mathrm{kpc}$; Barnes \& Hernquist 1996; Hopkins et al. 2012). This is well supported by the observations that AGNs are misaligned with the host galaxies (e.g., Kinney et al. 2000; Gallimore et al. 2006; Shen et al. 2010; Lagos et al. 2011). These conflicts reflect our poor understanding on the detailed processes in black hole fueling from the galaxy scale to the central disk scale. We await future observations to justify these conflicts and we content with our present assumption.

We consider only thin accretion disks with the disks' aspect ratio $H / R \ll \alpha$ to guarantee that the viscosity is strong enough to validate the Bardeen-Petterson effect (Papaloizou \& Lin 1995). Therefore, our approach only applies to the accretion disks with dimensionless accretion rate $\dot{M}_{\mathrm{h}} / \dot{M}_{\text {Edd }}$ at $10^{-2} \sim 1$, which are believed to correspond to the thin disk regime. Beyond this range, accretion disks transit to be thick and the Bardeen-Petterson effect may be weak or even disappear, as confirmed by recent magnetohydrodynamics (MHD) numerical simulations (e.g., Fragile 2009; Zhuravlev et al. 2014). However, the heuristic MHD simulations by McKinney et al. (2013) revealed a new "magneto-spin alignment" mechanism that works in thick disks with strong magnetic. Unlike the Bardeen-Petterson effect, this mechanism arises from the magnetic torque of the black hole's magnetosphere, which is already aligned with the black hole spin axis due to the framedragging forces. It is unknown yet how the spin evolves for thick warped disks and more investigations are highly worthwhile.

In conclusion, we study the alignments of black holes and their accretion disks by taking into account the finite sizes of disks and the continuous gas fueling. Our results show that, with fiducial values for the free parameters, the lifetime of gas fueling is crucial to the alignments/anti-alignments and therefore to the spin evolution. By applying our prescription to a series of accretion activities and assuming that the disk orientations are chaotically distributed over episodes, we compared our calculated spin distribution with the spin measurements 
through Iron $\mathrm{K} \alpha$ line fitting and through the radiative efficiency and made attempt to place constraints on the lifetime of gas fueling. Unfortunately, the spin measurements through these two techniques at this stage seems incompatible to allow us draw a firm conclusion. We find that generally a lifetime of gas fueling as $\Delta T_{\mathrm{f}}=10^{5} M_{8}^{-1} \mathrm{yr}$ can reproduce the spin distribution reported by the Iron $\mathrm{K} \alpha$ line fitting, whereas a lifetime as $\Delta T_{\mathrm{f}}=10^{5} M_{8}^{1.5} \mathrm{yr}$ can reproduce these constraint through the radiative efficiency. However, the later case seems consistent with the observed episodic lifetime as long as $\sim 10^{7} \mathrm{yr}$ for very luminous AGNs at high redshift, which plausibly harbor $>10^{9} M_{\odot}$ SMBHs. Since the lifetimes of gas fueling are linked to the episodic lifetimes of AGNs, we proposed that the episodic lifetimes should be regarded as a new ingredi- ent for the semi-analytic models of SMBH growth and spin evolution.

We thank the referee's suggestions that significantly improve the manuscript. LYR thanks Monica Colpi, Marta Volonteri and Enrico Barausse for useful discussions on black hole spin evolution, and the Institut d'Astrophysique de Paris where this work was completed. The research is supported by NSFC-11133006, 11173023, 11233003, and 11303026, the China-Israel nsfc-isf 1136114034, and the Strategic Priority Research Program - The Emergence of Cosmological Structures of the Chinese Academy of Sciences, grant No. XDB09000000.

\section{REFERENCES}

Adelberger, K. L., Steidel, C. C., Kollmeier, J. A., \& Reddy, N. A. 2006, ApJ, 637, 74

Benson, A. J., \& Bower, R. 2010, MNRAS, 405, 1573

Barnes, J. E., \& Hernquist, L. E. 1996, ApJ, 471, 115

Bardeen, J. M., \& Petterson, J. A. 1975, ApJ, 195, L65

Bardeen, J. M., Press, W. H., \& Teukolsky, S. A. 1972, ApJ, 178, 347

Brenneman, L. 2013, Measuring the Angular Momentum of Supermassive Black Holes (Springer: New York), arXiv: 1309.6334

Cantalupo, S., Lilly, S. J., \& Porciani, C. 2007, ApJ, 657, 135

Cao, X., \& Li, F. 2008, MNRAS, 390, 561

Chen, L., Wu, S., \& Yuan, F. 2009, MNRAS, 398, 1900

Collin, S., \& Zahn, J.-P. 1999, A\&A, 344, 433

Collin-Souffrin, S., \& Dumont, A. M. 1990, A\&A, 229, 292

Done, C., Jin, C., Middleton, M., \& Ward, M. 2013, MNRAS, 434, 1955

Czerny, B., Hryniewicz, K., Nikołajuk, M., \& Sagdowski, A. 2011, MNRAS, 415, 2942

Davis, S. W., \& Laor, A. 2011, ApJ, 728, 98

Dotti, M., Colpi, M., Pallini, S., Perego, A., \& Volonteri, M. 2013, ApJ, 762, 68

Dubois, Y., Pichon, C., Haehnelt, M., et al. 2012, MNRAS, 423, 3616

Dubois, Y., Volonteri, M., Silk, J., Devriendt, J., \& Slyz, A. 2014, MNRAS, 440,2333

Fanidakis, N., Baugh, C. M., Benson, A. J., et al. 2011, MNRAS, 410, 53

Fragile, P. C. 2009 , ApJ, 706, L246

Gallimore, J. F., Axon, D. J., O’Dea, C. P., Baum, S. A., \& Pedlar, A. 2006, AJ, 132,546

Gaspari, M., Ruszkowski, M., \& Oh, S. P. 2013, MNRAS, 432, 3401

Gonçalves, T. S., Steidel, C. C., \& Pettini, M. 2008, ApJ, 676, 816

Goodman, J. 2003, MNRAS, 339, 937

Ho, L. C., \& Kim, M. 2014, ApJ, 789, 17

Hopkins, P. F., Hernquist, L., Hayward, C. C., \& Narayanan, D. 2012, MNRAS, 425, 1121

Jiang, Y.-F., \& Goodman, J. 2011, ApJ, 730, 45

Jakobsen, P., Jansen, R. A., Wagner, S., \& Reimers, D. 2003, A\&A, 397, 891

Kawakatu, N., \& Wada, K. 2008, ApJ, 681, 73

Kendall, P., Magorrian, J., \& Pringle, J. E. 2003, MNRAS, 346, 1078

Kinney, A. L., Schmitt, H. R., Clarke, C. J., et al. 2000, ApJ, 537, 152

King, A. R., Lubow, S. H., Ogilvie, G. I., \& Pringle, J. E. 2005, MNRAS, 363,49

King, A. R., \& Pringle, J. E. 2006, MNRAS, 373, L90

King, A. R., Pringle, J. E., \& Hofmann, J. A. 2008, MNRAS, 385, 1621

Kirkman, D., \& Tytler, D. 2008, MNRAS, 391, 1457

Kollmeier, J. A., Onken, C. A., Kochanek, C. S., et al. 2006, ApJ, 648, 128

Kumar, P., \& Johnson, J. L. 2010, MNRAS, 404, 2170

Kumar, P., Narayan, R., \& Johnson, J. L. 2008, MNRAS, 388, 1729

Lagos, C. D. P., Padilla, N. D., \& Cora, S. A. 2009, MNRAS, 395, 625

Lagos, C. D. P., Padilla, N. D., Strauss, M. A., Cora, S. A., \& Hao, L. 2011, MNRAS, 414, 2148

Levin, Y. 2007, MNRAS, 374, 515

Lodato, G., \& Price, D. J. 2010, MNRAS, 405, 1212

Lodato, G., \& Pringle, J. E. 2006, MNRAS, 368, 1196

Lodato, G., \& Pringle, J. E. 2007, MNRAS, 381, 1287

Li, Y.-R., Ho, L. C., \& Wang, J.-M. 2011, ApJ, 742, 33

Li, Y.-R., Wang, J.-M., Cheng, C., \& Qiu, J. 2013, ApJ, 764, 16
Li, Y.-R., Wang, J.-M., \& Ho, L. C. 2012, ApJ, 749, 187

Lynden-Bell, D. 1969, Nature, 223, 690

Lubow, S. H., Ogilvie, G. I., \& Pringle, J. E. 2002, MNRAS, 337, 706

Martin, R. G., Pringle, J. E., \& Tout, C. A. 2007, MNRAS, 381, 1617

Martini, P. 2004, Coevolution of Black Holes and Galaxies, 169

McKinney, J. C., Tchekhovskoy, A., \& Blandford, R. D. 2013, Science, 339, 49

McLure, R. J., \& Dunlop, J. S. 2004, MNRAS, 352, 1390

McQuinn, M., \& Worseck, G. 2014, MNRAS, 440, 2406

Muñoz Marín, V. M., González Delgado, R. M., Schmitt, H. R., et al. 2007, AJ, 134, 648

Natarajan, P., \& Pringle, J. E. 1998, ApJ, 506, L97

Nayakshin, S., Power, C., \& King, A. R. 2012, ApJ, 753, 15

Nelson, R. P., \& Papaloizou, J. C. B. 1999, MNRAS, 309, 929

Nelson, R. P., \& Papaloizou, J. C. B. 2000, MNRAS, 315, 570

Nixon, C. J., King, A. R., \& Price, D. J. 2012, MNRAS, 422, 2547

Ogilvie, G. I. 1999, MNRAS, 304, 557

Paczynski, B. 1978, AcA, 28, 91

Papaloizou, J. C. B., \& Lin, D. N. C. 1995, ApJ, 438, 84

Perego, A., Dotti, M., Colpi, M., \& Volonteri, M. 2009, MNRAS, 399, 2249

Pringle, J. E. 1981, ARA\&A, 19, 137

Pringle, J. E. 1992, MNRAS, 258, 811

Reynolds, C. S. 2013, Space Sci. Rev., 81 (arXiv:1302.3260

Raimundo, S. I., Fabian, A. C., Vasudevan, R. V., Gandhi, P., \& Wu, J. 2012, MNRAS, 419,2529

Salpeter, E. E. 1964, ApJ, 140, 796

Scheuer, P. A. G., \& Feiler, R. 1996, MNRAS, 282, 291

Schmitt, H. R., Donley, J. L., Antonucci, R. R. J., et al. 2003, ApJ, 597, 768

Sesana, A., Barausse, E., Dotti, M., \& Rossi, E. M. 2014, arXiv:1402.7088

Shakura, N. I., \& Sunyaev, R. A. 1973, A\&A, 24, 337

Shankar, F., Weinberg, D. H., \& Miralda-Escudé, J. 2009, ApJ, 690, 20

Shen, S., Shao, Z., \& Gu, M. 2010, ApJ, 725, L210

Shen, Y., Greene, J. E., Strauss, M. A., Richards, G. T., \& Schneider, D. P. 2008, ApJ, 680, 169

Shlosman, I., \& Begelman, M. C. 1987, Nature, 329, 810

Sołtan, A. 1982, MNRAS, 200, 115

Thorne, K. S. 1974, ApJ, 191, 507

Trainor, R., \& Steidel, C. C. 2013, ApJ, 775, L3

Trakhtenbrot, B. 2014, ApJ, 789, L9

Ueda, Y., Akiyama, M., Hasinger, G., Miyaji, T., \& Watson, M. G. 2014, ApJ, 786, 104

Volonteri, M., Sikora, M., Lasota, J.-P., \& Merloni, A. 2013, ApJ, 775, 94

Volonteri, M., Sikora, M., \& Lasota, J.-P. 2007, ApJ, 667, 704

Wada, K., \& Norman, C. A. 2002, ApJ, 566, L21

Wang, J.-M., Chen, Y.-M., \& Zhang, F. 2006, ApJ, 647, L17

Wang, J.-M., Hu, C., Li, Y.-R., et al. 2009, ApJ, 697, L141

Wang, J.-M., Yan, C.-S., Gao, H.-Q., et al. 2010, ApJ, 719, L148

Worseck, G., Fechner, C., Wisotzki, L., \& Dall'Aglio, A. 2007, A\&A, 473, 805

Worseck, G., \& Wisotzki, L. 2006, A\&A, 450, 495

Wu, S., Lu, Y., Zhang, F., \& Lu, Y. 2013, MNRAS, 436, 3271

Zhuravlev, V. V., Ivanov, P. B., Fragile, P. C., \& Teixeira, D. M. 2014, arXiv: 1406.5515 